\title{
Alpha-Lipoic Acid: Therapeutic Potential in Diabetic Neuropathies
}

\author{
Victoria Serhiyenko*, Ludmila Serhiyenko, Mychailo Krasnyi and Alexandr Serhiyenko \\ Department of Endocrinology, Danylo Galytsky Lviv National Medical University, Ukraine
}

Submission: May 14, 2018; Published: May 30, 2018

*Corresponding author: Victoria Serhiyenko, Department of Endocrinology, Danylo Halytsky Lviv National Medical University, Ukraine, 69, Pekarska str., 79010, Ukraine, Tel: +38-322-76-94-96; Email: 965554@ukr.net; serhiyenkoa@gmail.com

Abstract

Diabetic neuropathy due to nerve damage caused by uncontrolled hyperglycemia is a common and serious complication strongly associated with diabetes mellitus. Its management focuses on long-term durable glycaemic control, multifactorial cardiovascular risk intervention, pathogenesis-oriented therapy. Clinical trials clearly suggest beneficial effects of $\alpha$-lipoic acid (ALA) consumption on lipid metabolism profile, endothelial activation, its anti-inflammatory, antithrombotic and antiatherosclerotic properties. We reviewed available evidence of the benefits of ALA administration, especially to patients with diabetic neuropathy, diabetic peripheral neuropathy, diabetic cardiovascular autonomic neuropathy, including effects on glucose and lipids metabolism parameters, thrombocyte aggregation state, endothelial function and antioxidant/ anti-inflammation properties.

Keywords: Alpha-lipoic acid; Diabetes mellitus; Diabetic neuropathy; Diabetic cardiovascular autonomic neuropathy; Lipids; Inflammation; Platelets; Endothelium; Heart rate variability

\section{Introduction}

Diabetes mellitus (DM) is a chronic metabolic disease with a high prevalence worldwide. DM and insulin resistance (IR) are associated with the development of cardiovascular and nervous diseases [1]. The development of these disorders reflects complex pathological processes in which the oxidative stress (OS) caused by reactive oxygen species (ROS) and reactive nitrogen species plays a pivotal role $[2,3]$.

The prevalence of neuropathy in diabetic patients is about $30 \%$, whereas up to $50 \%$ of patients will certainly develop neuropathy during the course of the disease [4,5]. The main pathophysiological pathway of diabetic neuropathy (DN) is associated with OS activation. Elevated intracellular levels of glucose lead to advanced glycation end-products formation and polyol pathway activation, resulting in subsequent formation of ROS [6]. Pathogenetic treatment of DN includes: balanced diet and physical activity; optimization of glycaemic control; treatment of dyslipoproteinemia; correction of metabolic abnormalities in myocardium; prevention and treatment of thrombosis; use of aldose reductase inhibitors, $\gamma$-linolenic acid, acetyl-L-carnitine, dihomo- $\gamma$-linolenic acid, antioxidants, first of all $\alpha$-lipoic acid (ALA), use of $\omega-3$ and $\omega-6$ polyunsaturated fatty acids, vasodilators, fatsoluble vitamin $\mathrm{B}_{1}$ (benfotiamine), aminoguanidine; substitutive therapy of growth factors and others $[3,7]$.

\section{Discussion}

Given the role of OS in diabetic neuropathy, diabetic peripheral neuropathy (DPN), diabetic cardiovascular autonomic neuropathy (CAN) progression, antioxidants such as acetyl-L-carnitine, taurine and ALA have been proven to be effective in preventing or delaying the onset of DPN [5,8-11]. Improvements in glucose disposal were also observed in patients with type 2 diabetes mellitus (T2DM) receiving ALA either i.v. or p.o. [12]. Also other studies findings represent ALA function in improving glycated haemoglobin A1c (HbA1c), lipid peroxidation, antioxidant enzymes and inflammatory markers. ALA has a function in decreasing damages caused by DN and inflammatory markers as a compound with strong antioxidant potential [13-16].

Five randomized double-blind placebo-controlled (RDBPC) trials, the Alpha-Lipoic Acid in Diabetic Neuropathy (ALADIN), Symptoms Of Diabetic Polyneuropathy (SYDNEY), Oral Pilot (ORPIL), SYDNEY 2, and ALADIN III studies, comprising a total of 1160 people were selected. The study populations included individuals ranging in age from 18 to $74 \mathrm{yr}$ with T1DM and T2DM. Mean body mass indices ranged from 27.7 to $30.9 \mathrm{~kg} / \mathrm{m}^{2}$. On average, initial HbA1c was $<12 \%$ and average DM duration ranged from 10.4 to $15.1 \mathrm{yr}$, while that of diabetic sensorimotor 


\section{Current Research in Diabetes \& Obesity Journal}

polyneuropathy (DSPN) ranged from 2.8 to $5 \mathrm{yr}$. The five studies evaluated the effectiveness of ALA on DSPN using three different types of drug administration. Two studies evaluated the role of i.v. ALA compared with placebo. The parenteral treatment was given to separate intervention groups in doses of $100 \mathrm{mg}, 600$, and $1200 \mathrm{mg}$ q.d. A total of 14 treatments were given over $3 \mathrm{wks}$ in both studies $[17,18]$. Two of them examined the effects of p.o. ALA administration relative to control patients with DSPN. Oral treatment was given to individual groups at total q.d. of $600 \mathrm{mg}$, $1200 \mathrm{mg}$, and $1800 \mathrm{mg}$. Duration of treatment ranged from 3 to 5 wks $[17,19]$. One trial included a combination of parenteral and p.o. ALA given sequentially. Combination therapy included schedules of ALA $600 \mathrm{mg}$ iv daily for 3 wks prior to $1800 \mathrm{mg}$ p.o. daily for $6 \mathrm{mos}$ and $600 \mathrm{mg}$ i.v. daily prior to p.o. placebo for $6 \mathrm{mos}$ versus iv placebo treatment followed by p.o. placebo treatment. The total study duration was 7 mos $[18,20]$. Treatment with ALA $600 \mathrm{mg}$ i.v. q.d. for 3 wks represents a well-tolerated and effective therapy for DSPN. Similarly, an p.o. dose of $600 \mathrm{mg}$ q.d. administered for up to $5 \mathrm{wks}$ could offer benefits in symptoms and signs of DSPN without significant side effects. Whereas a single study using sequential parenteral and p.o. therapy failed to show a significant effect, the preponderance of evidence supports the effectiveness of ALA in treating the symptoms and signs of DSPN [18]. Benefits of p.o. treatment with ALA were studied in SYDNEY 2. A 5wks trial showed that p.o. treatment with ALA for 5 wks improved neuropathic symptoms and deficits in patients with DPN, and an p.o. dose of $600 \mathrm{mg}$ once daily provide the optimum risk-to-benefit ratio while effect on electrophysiological parameters was not reported [5].

Two recent meta-analysis evaluate the use of ALA in DN. One of them, included 1.258 diabetic patients treated with $600 \mathrm{mg}$ of ALA i.v. for three wks, concluded that pain, numbness and burning decreased significantly with ALA in comparison to placebo. Considering the components of neuropathy impairment score Lower Limb (NIS-LL) an improvement was noted in pin-prick, touch pressure and ankle reflexes. This meta-analysis also pointed out some relevant aspects for conducting future trials to evaluate the benefits of ALA on DN as follows: homogeneity of the studied patients; duration of the trial; end-points with less variability and finally considering the slowing progression of DN the end point must have to exclude the latter and address improvement $[21,22]$. The other meta-analysis included 653 diabetic patients treated with different doses of ALA p.o. (two studies) or i.v. (two studies) for three (3 studies) to $5 \mathrm{wks}$ (one study) concluded that total symptoms score (TSS) decreased significantly but only in the i.v. study the TSS decrease more than $30 \%$ which was considered to be clinically significant. Recently, a non-randomized, openlabel and prospective study has shown an improvement in pain and eletroneurographic parameters, mainly in sensory nerve conduction, among 50 patients with DSPN treated with a new p.o. formulation combining ALA $400 \mathrm{mg} / \mathrm{g}$.d. and superoxide dismutase 140IU/g.d. for four mos [22-24].
The results of meta-analysis provide evidence that treatment with ALA (300-600 mg/day i.v. for 2-4 wks) is safe and that the treatment can significantly improve both nerve conduction velocity and neuropathic symptoms [25]. Finally, it is important to mention the NATHAN 1 Trial (Neurological Assessment of Thioctic Acid in Diabetic Neuropathy), a multicenter study which used 600mg of ALA q.d. for four yr with NIS-LL + seven neurophysiologic tests as primary outcome. In this study after a four-yr treatment with ALA in mild-to-moderate DSPN did not influence the primary composite end point but resulted in a significant clinical improvement and prevention of progression of neuropathic impairments. As the primary composite end point did not deteriorate in placebo-treated subjects, secondary prevention of its progression by ALA according to the trial design was not feasible. All these latter studies concluded that the usual dose of $600 \mathrm{mg}$ is safe and well tolerated with mainly dose dependent gastrointestinal adverse events. Moreover, with one exception (sural latency), all these studies did observe improvement in electrophysiological tests [22,26].

A RDBPC study with 30 patients with T2DM evaluated glycaemic control and endothelial responses to i.v. acetylcholine (endothelium-dependent) and nitrate (endotheliumindependent) in order to evaluate the forearm blood flow before and after the use of $600 \mathrm{mg}$ of ALA i.v. for three wks. A decrease in $\mathrm{HbA1c}$, total cholesterol (TC) and triglycerides (TG) levels were observed in both groups. However only the ALA treated patients showed an improvement in the endothelium dependent vasodilation $[22,27]$.

The Irbesartan and Lipoic Acid in Endothelial Dysfunction (ISLAND) clinical trial has examined ALA as a potential remedy for endothelial dysfunction. This trial was a RDBPC study comparing ALA to irbesartan, an angiotensin II receptor antagonist used mainly for the treatment of hypertension. Results showed that the p.o. administration of ALA (300mg/q.d. for $4 \mathrm{wks}$ ) and/or irbesartan (150mg/q.d. for $4 \mathrm{wks}$ ) to 15 patients with metabolic syndrome (MetS) in each group improved endothelial-dependent flow-mediated vasodilation, which was measured by using the noninvasive brachial artery reactivity test $[12,28]$. The ISLAND trial showed a $15 \%$ significant decrease in serum interleukin (IL)6 levels following 4 wks of supplementation with ALA (300mg/ day). This finding may prove the anti-inflammatory effects of ALA, as IL-6 is a recognized marker of inflammation in coronary atherosclerotic plaques, and also regulates the expression of other inflammatory cytokines, such as IL-1 and tumor necrosis factoralpha. However, the body of evidence is currently too limited and could not be considered as conclusive [12,28].

A RDBPC study (DEKAN) was conducted in patients with T2DM with CAN using $800 \mathrm{mg}$ of ALA q.d. for 16wks. Cardiovascular autonomic tests (CART's) and heart rate variability (HRV) were evaluated before and after treatment. The intervention with ALA resulted in improvement of some HRV parameters: root mean square successive difference (RMSSD) and power spectrum in 


\section{Current Research in Diabetes \& Obesity Journal}

low frequency band (LF). No difference in overall symptoms was observed [29].

We have carried out two studies to analyze the effectiveness of ALA. In the first one we have analyzed the effectiveness of ALA on activity of acethylcholinesterase (ACE) in erythrocytes membrane, superoxide dismutase (SOD), glutation peroxidase (GPO) and catalase (CAT) in RBC's, osmotic stability, levels of $\mathrm{Na}^{+}$and $\mathrm{K}^{+}$in erythrocytes, TC, low (LDL-C), and high density lipoprotein-cholesterol (HDL-C), TG in T2DM patients with CAN. 67 patients were allocated in two treatment groups. Thioctic acid was administered i.v. at a q.d. dose of $600 \mathrm{mg}$, during $2 \mathrm{wks}$, and single q.d of $600 \mathrm{mg}$ p.o during the next 2 mos. It was defined that ACE activity increases in RBC's membrane what testifies about the reconstruction of membranes, penetration increasing of them, especially for $\mathrm{Na}^{+}$and $\mathrm{K}^{+}$(confirmed by increased levels of $\mathrm{Na}^{+}$and $\mathrm{K}^{+}$in erythrocytes). High level of TC, LDL-C, TG (p $<0.05$ ) and depressed enzymes activity were diagnosed in all of them. At the end of 2mos TG and LDL-C levels were decreased more significantly in patients from treatment group. Also, we observed more significantly improve of CART's. ALA in dose of $600 \mathrm{mg}$ q.d. during $2 \mathrm{mos}$ increases activity of SOD ( $\mathrm{p}<0.001$ ), GPO ( $<<0.001)$ and CAT ( $<<0.05)$, osmotic stability in RBC's, what testify about the increase of antioxidant defense and stabilization of lipids bilayer in erythrocytes membrane. So, ALA promotes to decrease the activity of ACE in RBC's membrane, what testify about the tendention to normalize penetration of membranes, especially for $\mathrm{Na}^{+}$and $\mathrm{K}^{+}[30,31]$. To the second one were included 49 patients with T2DM and CAN. All patients were randomized to receive either a daily i.v. (2wks) and then p.o. dose of $600 \mathrm{mg}$ ALA $(n=32)$ or placebo $(n=17)$ during 2 mos. CAN assessed by CART's, HRV, and QTc interval parameters disturbances. Analysis of aggregatory curves shows that platelets in patients with CAN began to aggregate earlier and the speed $(p<0.001)$ and the stage of aggregation $(p<0.01)$ increase. Obtained results could witness about increase in platelet sensitivity towards thrombin and ADP in T2DM patients with CAN. There was an increase in ${ }^{125}$ I-thromboxane $\mathrm{B}_{2}\left(\mathrm{TXB}_{2}\right)$ level $(\mathrm{p}<0.001)$, decreases of ${ }^{125}$ I-6ketoprostaglandin $\mathrm{F}_{1 \alpha}\left(6-\right.$ ketoPGF$\left._{1 \alpha}\right)$ in plasma blood, activities of SOD, GPO and CAT in platelets and RBC's, content of glutathione (GSH) ( $p$ <.001) simultaneously. After 2 mos of treatment with ALA the decrease of degree and speed of thrombocytes aggregation was marked. Simultaneously, concentration of 6-ketoPGF ${ }_{1 \alpha^{\prime}}$ GSH and activity of SOD, GPO were increased and the content of $\mathrm{TXB}_{2}$ was considerably reduced $(\mathrm{p}<0.01)$. The above-stated changes were accompanied by positive dynamics of tool and functional samples permitting to troubleshoot a cardiac pathology, and, especially, CAN in patients with T2DM. So, prescription of ALA was associated with improvement of antioxidant status and platelet aggregation parameters in T2DM patients with CAN [32]. The administration of ALA to T2DM patients with subclinical stage of CAN positively influences parameters of HRV, in particular increase of RMSSD ( $p$ <.001), LF ( $p<0.001)$ and high frequency
(HF) $(\mathrm{p}<0.001)$ parameters was observed. The obtained data can testify the increase of nervous impulse realization rate in visceral parasympathetic and sympathetic fibers [33].

A prospective, RDBPC study was performed in 44 patients with T1DM presenting any diabetes-related chronic complication (mild non-proliferative retinopathy or microalbuminuria and the presence of CAN was defined by an alteration of positron emission tomography with normal CART's. Patients were submitted to triple antioxidant therapy with the objective to target different pathways of OS damage: inhibition of xanthine oxidase by allopurinol $300 \mathrm{mg} /$ q.d., inhibition of OS by ALA, $600 \mathrm{mg} / \mathrm{b}$.i.d. and inhibition of poly (ADP-ribose). In this study no improvement was found in all parameters of autonomic function analysis as well as in urinary levels of isoprostanes, a marker of OS [34]. Clinically, ALA administration (in combination with acetyl-L-carnitine) showed some promise as an antihypertensive therapy by decreasing systolic pressure in high blood pressure patients and subjects with the MetS. In contrast, the administration of ALA $(300 \mathrm{mg} /$ day for $4 \mathrm{wks}$ ) to patients with the MetS had no significant effect on blood pressure compared to placebo group [12,22,34].

The combination of ALA and vitamin B complex was effective in improving nerve function parallel to symptoms improvement [5]. There was a significant reduction after $12 \mathrm{wks}$ of supplementation in vitamin B complex plus ALA group using the Michigan Neuropathy Screening Instrument (MNSI) questionnaire and not in vitamin B complex group. Study demonstrated that 12 out of 16 patients in vitamin B complex plus ALA group showed improvement in at least one property measured compared with only 6 out of 16 in vitamin B complex group. The influence of the combination on the progression of DM with regards to IR and lipid profile showed no advantage over vitamin B complex alone [5]. ALA has been used in 94 patients with T2DM in a RDBPC study which has tested three antioxidants for three mos in association with metformin and glimeperide: ALA, 300mg/q.d., ecosapentanoic acid, $180 \mathrm{mg}$ plus docohexaenoic acid, $120 \mathrm{mg} /$ q.d. omega 3 fatty acids and vitamin E $400 \mathrm{mg} / \mathrm{q}$.d. Although an improvement in $\mathrm{HbA1c}$, weight and waist have been observed with ALA, omega 3 fatty acids gave the better results concerning weight loss and glycaemic control [22].

\section{Conclusion}

ALA has been widely prescribed drug for treatment and prevention of chronic diabetic complications since it affects the main pathogenesis links. The many unique properties of ALA, namely prevention of beta-cell destruction, enhancement of glucose uptake, antioxidant effects, inhibition of glycation reactions, restoration of vitamins levels, improvement of neurons function and conduction has been shown in a number of experimental and clinical trials. However, further randomized, double-blind, placebo-controlled trials of larger scale and longer duration are necessary to strengthen existing data and provide greater insight into the long-term efficacy of ALA. 


\section{Current Research in Diabetes \& Obesity Journal}

\section{Conflict of Interest}

The authors have declared no financial/commercial conflict of interest.

\section{References}

1. American Diabetes Association (2017) Standards of medical care in diabetes -2017. Diabetes Care 40(1S): S1-S132.

2. Pitocco D, Tesauro M, Alessandro R, Ghirlanda G, Cardillo C (2013) Oxidative stress in diabetes: implications for vascular and other complications. Int J Mol Sci 14(11): 21525-21550.

3. Serhiyenko VA, Serhiyenko AA (2018) Cardiac autonomic neuropathy: Risk factors, diagnosis and treatment. World J Diabetes 9(1): 1-24.

4. Callaghan BC, Cheng HT, Stables CL, Smith AL, Feldman EL (2012) Diabetic neuropathy: Clinical manifestations and current treatment Lancet Neurol 11(6): 521-534.

5. Boghdadi MA, Afify HE, Sabri N, Makboul K, Elmazar M (2017) Comparative study of vitamin B complex combined with alpha lipoic acid versus vitamin B complex in treatment of diabetic polyneuropathy in type 2 diabetic patients. Clin Exp Pharmacol 7: 241.

6. Hosseini A, Abdollahi M (2013) Diabetic neuropathy and oxidative stress: Therapeutic perspectives. Oxid Med Cell Longe 2013: 1-15.

7. Vinik AI, Nevoret ML, Casellini C, Parson H (2013) Diabetic neuropathy. Endocrinol Metab Clin North Am 42(4): 747-787.

8. Ziegler D, Low PA, Litchy WJ, Boulton AJ, Vinik AI, et al. (2011) Efficacy and safety of antioxidant treatment with $\alpha$-lipoic acid over 4 years in diabetic polyneuropathy: the NATHAN 1 trial. Diabetes Care 34(9) 2054-2060.

9. Vallianou N, Evangelopoulos A, Koutalas P (2009) Alpha-lipoic acid and diabetic neuropathy. Rev Diabet Stud 6(4): 230-236.

10. Golbidi S, Badran M, Laher I (2011) Diabetes and alpha lipoic acid. Front Pharmacol 2: 69.

11. Shakher J, Stevens MJ (2011) Update on the management of diabetic polyneuropathies. Diabetes Metab Syndr Obes 4: 289-305.

12. Shay KP, Moreau RF, Smith EJ, Smith AR, Hagen TM (2009) Alpha-lipoic acid as a dietary supplement: Molecular mechanisms and therapeutic potential. Biochim Biophys Acta 1790(10): 1149-1160.

13. Ibrahimpasic K (2013) Alpha lipoic acid and glycaemic control in diabetic neuropathies at type 2 diabetes treatment. Med Arch 67(1): 7-9.

14. Morakinyo AO, Awobajo FO, Adegoke OA (2013) Effects of alpha lipoic acid on blood lipids, renal indices, antioxidant enzymes, insulin and glucose level in streptozotocin-diabetic rats. Biol Med 5: 26-33.

15. Feng B, Yan XF, Xue JL, Xu L, Wang H (2013) The protective effects of $\alpha$-lipoic acid on kidneys in type 2 diabetic Goto-Kakisaki rats via reducing oxidative stress. Int J Mol Sci 14(4): 6746-6756.

16. Ahmadvand H, Jamor P (2017) Effects of alpha lipoic acid on level of NO and MPO activity in diabetic rats. Ann Res Antioxid 2(2): E04.

17. Ziegler D, Ametov A, Barinov A, Dyck PJ, Gurieva I, et al. (2006) Oral treatment with alpha-lipoic acid improves symptomatic diabetic polyneuropathy: the SYDNEY 2 trial. Diabetes Care 29(11): 2365-2370.

18. McIlduff CE, Rutkove SB (2011) Critical appraisal of the use of alpha lipoic acid (thioctic acid) in the treatment of symptomatic diabetic polyneuropathy. Ther Clin Risk Manag 7: 377-385.

19. Ruhnau KJ, Meissner HP, Finn JR, Reljanovic M, Lobisch M, et al. (1999) Effects of 3-week oral treatment with the antioxidant thioctic acid (alpha-lipoic acid) in symptomatic diabetic polyneuropathy. Diabet Med 16(12): 1040-1043.

20. Ziegler D, Hanefeld M, Ruhnau KJ, Meissner HP, Lobisch M, et al. (1995) Treatment of symptomatic diabetic peripheral neuropathy with the anti-oxidant alpha-lipoic acid. A 3-week multicentre randomized controlled trial (ALADIN Study). Diabetologia 38(12): 1425-1433.

21. Ziegler D, Nowak H, Kempler P, Vargha P, Low PA (2004) Treatment of symptomatic diabetic polyneuropathy with the antioxidant alphalipoic acid: a meta-analysis. Diabet Med 21(2): 114-121.

22. Gomes MB, Negrato CA (2014) Alpha-lipoic acid a pleiotropic compound with potential therapeutic use in diabetes and other chronic diseases. Diabetol Metab Syndr 6(1): 80.

23. Mijnhout GS, Kollen BJ, Alkhalaf A, Kleefstra N, Bilo HJ (2012) Alpha lipoic acid for symptomatic neuropathy in patients with diabetes: A meta-analysis of randomized controlled trials. Int J Endocrinol 2012 456279

24. Bertolloto F, Massome A (2012) Combination of alpha lipoic acid and superoxide dismutase leads to physiological and symptomatic improvements in diabetic neuropathy. Drugs R D 12(1): 29-34.

25. Han T, Bai B, Liu W, Hu Y (2012) A systematic review and meta-analysis of $\alpha$-lipoic acid in the treatment of diabetic peripheral neuropathy. Eur J Endocrinol 167(4): 465-471.

26. Ziegler D, Low PA, Litchy WJ, Boulton AJ, Vinik AI, et al. (2011) Efficacy and safety of antioxidant treatment with $\alpha$-lipoic acid over 4 years in diabetic polyneuropathy: the NATHAN 1 trial. Diabetes Care 34(9): 2054-2060.

27. Heinisch BB, Francesconi M, Mittermayer F, Schaller G, Gouya G, et al. (2010) Alpha-lipoic acid improves vascular endothelial function in patients with type 2 diabetes: a placebo-controlled randomized trial. Eur J Clin Invest 40(2): 148-154.

28. Sola S, Mir MQ Cheema FA, Khan Merchant N, Menon RG, et al. (2005) Irbesartan and lipoic acid improve endothelial function and reduce markers of inflammation in the metabolic syndrome: results of the Irbesartan and Lipoic Acid in Endothelial Dysfunction (ISLAND) study. Circulation 111(3): 343-348.

29. Ziegler D, Schatz H, Conrad F, Gries FA, Ulrich H, et al. (1997) Effects of treatment with the antioxidant alpha-lipoic acid on cardiac utonomic neuropathy in NIDDM patients. A 4-month randomized controlled multicenter trial (DEKAN Study). Deutsche Kardiale Autonome Neuropathie. Diabetes Care 20(3): 369-373.

30. Serhiyenko VA, Serhiyenko AA, Petrychka OP, Serhiyenko LM (2003) Effect of $\alpha$-lipoic acid on lipid and the erythrocyte metabolism in type 2 diabetic patients with cardiovascular autonomic neuropathy. Diabetes Metab 29(2): 2412.

31. Serhiyenko AA, SerhiyenkoVA, Petrychka OP (2003) Effect of $\alpha$-lipoic acid on lipid and the erythrocyte metabolism in NIDDM patients with cardiovascular autonomic neuropathy. J Peripher Nerv Syst 8(3): 201.

32. Serhiyenko AA, Petrychka OP, Serhiyenko LM, Serhiyenko VA (2003) The effects of lipoic acid on antioxidant status and platelet aggregation in type 2 diabetic patients with cardiovascular autonomic neuropathy. Diabetes Metab 29(2): 2411.

33. Petrychka OP, Serhiyenko AA, Serhiyenko VA (2003) Effects of $\alpha$-lipoic acid on heart rate variability, QTc interval parameters, and antioxidant status in type 2 diabetic patients with cardiovascular autonomic neuropathy. J Peripher Nerv Syst 8(3): 200-201.

34. Pop Busui R, Stevens MJ, Raffel DM, White EA, Mehta M, et al. (2013) Effects of triple antioxidant therapy on measures of cardiovascular autonomic neuropathy and on myocardial blood flow in type 1 diabetes: a randomised controlled trial. Diabetologia 56(8): 1835-1844. 
This work is licensed under Creative Commons Attribution 4.0 Licens

DOI: 10.19080/CRDOJ.2018.07.555713
Your next submission with Juniper Publishers will reach you the below assets

- Quality Editorial service

- Swift Peer Review

- Reprints availability

- E-prints Service

- Manuscript Podcast for convenient understanding

- Global attainment for your research

- Manuscript accessibility in different formats

( Pdf, E-pub, Full Text, Audio)

- Unceasing customer service

Track the below URL for one-step submission https://juniperpublishers.com/online-submission.php 